\title{
Effect of Training Types Using Recumbent Cycle Ergometer on Ankle Strength in Healthy Male Subjects
}

\author{
Ho-Youl Ryu', In-Cheol Jeon², Ki-Song Kim² \\ ${ }^{1}$ Rehabilitation Medical Team, Yong-In Severance Hospital, Yonsei University Health System, Yongin, Republic of Korea; ${ }^{2}$ Department of Physical \\ Therapy, College of Life and Health Science, Hoseo University, Research Institute for Basic Sciences, Asan, Republic of Korea
}

Purpose: The purpose of this study was to investigate the exercise effect of two types of training with a recumbent cycle ergometer on ankle muscle strength (dorsiflexor strength, DFS; dorsiflexor strength/weight, DFS/kg; plantar flexor strength, PFS; and plantar flexor strength/weight, PFS/kg) in healthy male subjects.

Methods: Twenty-three healthy males $(27.91 \pm 8.66 \mathrm{yr})$ were randomly allocated into two groups (high-intensity interval training (HIIT), and aerobic exercise training (AET) after the first measurement. The subjects were trained for 24 sessions (40 min/rep, three times/week) and ankle strength was measured for a second time. Two-way mixed model analysis of variance (ANOVA) was used to identify significant differences between changes in ankle muscle strength between before and after training (within factors) in the HIIT and AET groups (between factors). The statistical significance level was set at $\alpha=0.05$.

Results: In both HIIT and AET groups, all variables of ankle muscle strength were significantly increased after training compared to before training $(p=0.001)$. However, there were no differences in all variables of ankle strength between the HIIT and AET group $(p>0.05)$.

Conclusion: Both types (HIIT and AET) of recumbent cycle exercise training could be effective training methods to increase ankle muscle strength in healthy individuals, and the HIIT type with high intensity and low frequency pedaling could be recommended more to strengthen ankle muscles.

Keywords: Aerobic exercise, Ankle strength, High-intensity interval training, Recumbent cycle

\section{INTRODUCTION}

The recumbent cycle ergometer is used in a supine position, which is different from conventional cycling exercise, which is done in an upright or slightly forward-tilted sitting position..$^{1.5}$ Recently, studies on the exercise effect of recumbent cycle exercise, which involves pedaling in a recumbent position, have been conducted. ${ }^{1-5}$ When exercising at the same intensity, the recumbent position reduced the rate pulse product compared to an upright or slightly forward-tilted sitting position, ${ }^{2,3}$ and increased the thresholds of joint pain and chest pain felt when the maximum ability is exerted during exercise. ${ }^{4}$ Therefore, the recumbent cycle ergometer in the recumbent position could be effective in improving functional abilities and exercise capacity. Also, exercise intensity can be increased with the recumbent cycle ergometer because of these advantages of the recumbent position. $^{2,3}$
There are two training methods with the recumbent cycle ergometer: high-intensity interval training (HIIT) and aerobic exercise training (AET). HIIT consists of pedaling exercise with a high load and a low load of the maximum workload (MWL) for a certain period with a fixed speed rate. ${ }^{2,3}$ In contrast, AET consists of pedaling exercise with a constant mid load of the MWL for a certain period with a fixed speed rate. ${ }^{2,3}$ Therefore, the HIIT method may contribute to muscle strength of the lower extremities, while the AET method may contribute to muscle endurance of the lower extremities. ${ }^{3}$

Cycle exercises for a certain period can be effective in the improvement of muscle strength and endurance around the hip, knee and ankle joints. ${ }^{3.4}$ Since ankle muscle strength is reported to be a major factor that affects dynamic balance, walking speed, and the incidence of falls in various populations, ${ }^{67}$ the improvement of ankle muscle strength can contribute to the improvement of the quality of life and the reduction of potential in-
Received Nov 16, 2021 Revised Dec 16, 2021

Accepted Dec 20, 2021

Corresponding author Ki-Song Kim

E-mail kskim68@hoseo.edu
Copylight ( 2021 The Korean Society of Physical Therapy

This is an Open Access article distribute under the terms of the Creative Commons Attribution Non-commercial License (https:// creativecommons.org/license/by-nc/4.o.) which permits unrestricted non-commercial use, distribution, and reproduction in any medium, provided the original work is properly cited. 
juries. Studies on the exercise effect of cycling in an upright or slightly forward-tilted sitting position have been common. ${ }^{89}$ Indoor cycling exercise is widely used to improve muscle weakness because the joint range of motion and muscle contraction patterns during exercise are constant and repetitive. ${ }^{8}$ However, there are almost no studies on the effects of recumbent cycle ergometer training on ankle muscle strength according to the two recumbent cycle ergometer training methods, HIIT and AET.

Therefore, it is necessary to examine how the two recumbent cycle ergometer training methods affect ankle muscle strength in healthy general subjects. This study aims to investigate which of the two recumbent cycle ergometer training methods is more effective for changes in the ankle muscle strength of healthy subjects and recommend a recumbent cycle ergometer training method suitable for subjects' physical characteristics. The hypotheses of this study are first "two recumbent cycle ergometer training methods, both HIIT and AET, will contribute to the improvement of ankle muscle strength and ratio after exercises compared to before exercises" and second "The subjects assigned to the groups for the two types of training programs will have different improvements in ankle muscle strength."

\section{METHODS}

\section{Subjects}

For this study, healthy male subjects with no experience in recumbent cycle ergometer training at normal times were recruited. ${ }^{10,11}$ Twenty-four university students and school personnel who were currently attending or working at a university in Chungnam applied. Written consent was obtained from those subjects who expressed their intention to participate in the study after an explanation of the study. The study was received with approval from the Hoseo University Institutional Review Board (1041231-211019-HR-
133-02). Those with metabolic diseases such as diabetes or endocrine disease, or disc or musculoskeletal pain disease were excluded. In addition, those who used exercise facilities or received exercise training for fitness within 6 months of the time of participation in this study were excluded. Out of the total 24 applicants, one who complained of discomfort during recumbent training was excluded, so that 23 applicants finally participated in the experiment. The participants were randomly assigned to the HIIT or AET group.

There was no significant difference between the two groups in any of the variables, which were the general characteristics of subjects in the groups, dorsiflexor strength (DFS), plantar flexor strength (PFS), the ratio of dorsiflexor strength to weight (DFS/kg), and the ratio of plantar flexor strength to weight (PFS/kg) (Table 1).

\section{Measurements}

\section{1) Ankle muscle strength test}

Twenty-three healthy subjects were randomly divided into two groups according to recumbent cycle exercise training programs, and DFS and PFS before training were measured using Micro FET (Hoggan Health Industries Inc, West Jordan, USA) (Figure 1). ${ }^{12}$

\section{2) Recumbent Incremental Load Maximum Motion Test}

In order to determine the standardized exercise training intensity considering the differences in body weight and physical strength among the subjects, a built-in incremental load maximal exercise test was performed with tablet computer software connected to the recumbent cycle. The incremental load maximum exercise test is a ramp protocol in which exercise begins at a speed of 60 RPM under a load of 10 watts and the load is automatically increased by 10 watts every minute. The exercise test is terminated in cases where the subject is exhausted and stops exercise or fails to maintain a speed of 60 RPM or higher for more than 10 seconds despite

Table 1. Comparison of subject characteristics between HIIT group and AET group

\begin{tabular}{|c|c|c|c|c|}
\hline & \multicolumn{2}{|c|}{ Mean $\pm S D$} & \multirow{2}{*}{$\mathrm{t}$} & \multirow{2}{*}{$p$} \\
\hline & HIIT $(n=12)$ & $\operatorname{AET}(n=11)$ & & \\
\hline Age (yr) & $29.3 \pm 10.4$ & $26.4 \pm 6.3$ & 0.815 & 0.424 \\
\hline Height (cm) & $173.6 \pm 5.1$ & $174.6 \pm 5.1$ & -0.453 & 0.655 \\
\hline Weight (kg) & $73.8 \pm 7.1$ & $82.6 \pm 13.2$ & -1.316 & 0.202 \\
\hline BMI (kg/m2) & $24.5 \pm 2.1$ & $27.0 \pm 3.7$ & -1.172 & 0.254 \\
\hline DFS (kg) & $34.81 \pm 5.43$ & $37.74 \pm 8.46$ & 1.024 & 0.318 \\
\hline $\mathrm{DFS} / \mathrm{kg}$ & $0.48 \pm 0.09$ & $0.46 \pm 0.07$ & 1.767 & 0.092 \\
\hline PFS (kg) & $27.91 \pm 2.02$ & $32.63 \pm 6.69$ & 0.979 & 0.339 \\
\hline $\mathrm{PFS} / \mathrm{kg}$ & $0.38 \pm 0.04$ & $0.39 \pm 0.08$ & 1.572 & 0.131 \\
\hline
\end{tabular}

HIIT: high intensity interval training, AET: aerobic exercise training, BMl: body mass index, DFS: dorsi flexors strength, PFS: plantar flexors strength, SD: standard deviation. 


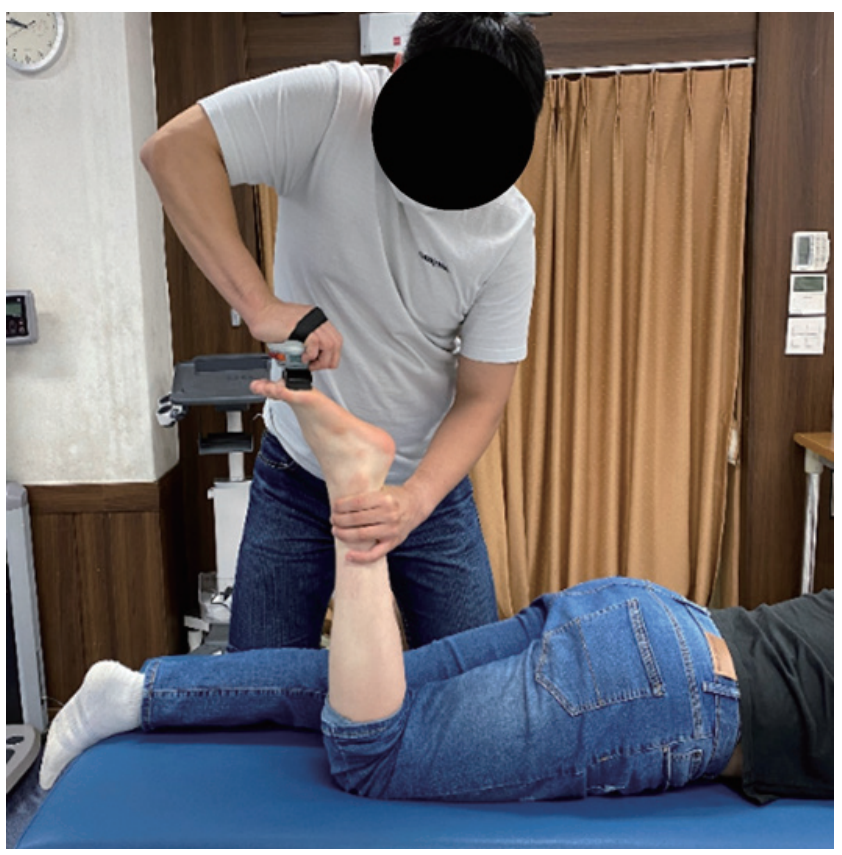

Figure 1. Test of ankle strength.

maximum effort. The load at the end point was recorded as the MWL, and the exercise training intensity was determined as the percentage (\%) of the MWL.

\section{Procedure}

All subjects in the two groups participated in recumbent cycle exercise training with programs consisting of 40 minutes per session, three sessions a week for a total of 24 sessions (Figure 2). The compositions of the exercise training programs are as follows.

\section{1) Warm-up exercise}

Light walking and simple joint exercise were performed for 5 minutes before the start of the main exercise.

\section{2) Main exercise}

Recumbent cycle exercise consisting of 40 minutes was performed. The detailed compositions of exercise training programs of individual groups are as follows.

(1) The HIIT group performed pedaling exercise in which a high load (100\% MWL, 20 seconds) and a low load (10\% MWL, 40 seconds) were automatically repeated for 40 minutes at a fixed speed of 20 RPM of the recumbent cycle.

(2) The AET group performed a pedaling exercise in which a load of 40\% MWL was provided for 40 minutes at a fixed speed of 60 RPM

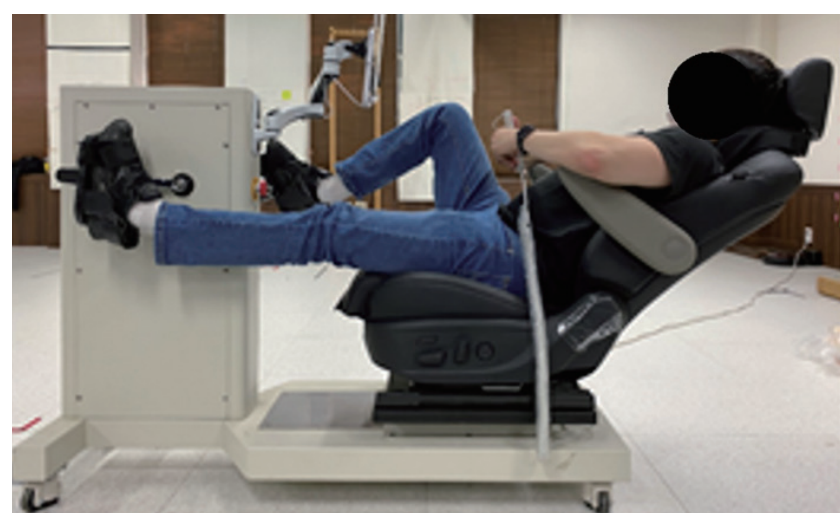

Figure 2. Recumbent cycle training.

Table 2. Exercise Intensity and Energy Expenditure/minute

\begin{tabular}{lcc}
\hline & El/min $(\% \mathrm{MWL})$ & $\mathrm{EE} / \mathrm{min}(\% \mathrm{MWL})$ \\
\hline HIIT & $(100 \% \mathrm{MWL} \times 20 \mathrm{sec})+$ & $(100 \times 1 / 3)+$ \\
& $(10 \% \mathrm{MWL} \times 40 \mathrm{sec})$ & $(10 \times 2 / 3)=40 \%$ \\
AET & $40 \% \mathrm{MWL} \times 60 \mathrm{sec}$ & $40 \times 1=40 \%$ \\
\hline
\end{tabular}

HIIT: high intensity interval training, AET: aerobic exercise training, El: exercise intensity, MWL: maximum workload, EE: energy expenditure.

of the recumbent cycle.

(3) Cool-down exercise: After 40 minutes of the main recumbent cycle exercise, light walking and cool-down stretching exercise were performed for 5 minutes.

Although the exercise programs of the two groups designed in this study differed in pedaling speeds and applied loads, the energy consumption per minute was designed to be the same, so that there was no difference in total energy consumption between both groups (Table 2).

\section{Data Analysis}

All the data measured in this study were analyzed using the SPSS ver. 25.0 program. A one-sample Kolmogorov-Smirnov test was used to ensure a normal distribution of the data. Descriptive statistics were used to analyze the general characteristics of the subjects. Two-way mixed model analysis of variance (ANOVA) was employed to identify significant differences between changes in ankle muscle strength and ratio between before and after training (within factors) in each group (between factors). If a significant interaction occurred between the between and within factors, the simple effect was investigated using Bonferroni correction ( $\alpha=0.05 / 4=0.0125)$. The statistical significance level was set at $\alpha=0.05$.

\section{RESULTS}

The 23 subjects assigned to the recumbent cycle exercise training pro- 
Table 3. Comparison of changes in ankle strength between two training groups at the time of measurements

\begin{tabular}{|c|c|c|c|c|}
\hline \multirow{3}{*}{ Variables } & \multicolumn{2}{|c|}{ Mean $\pm S D$} & \multirow{3}{*}{$\mathrm{F}$} & \multirow{3}{*}{$p$} \\
\hline & \multicolumn{2}{|c|}{ Training Program } & & \\
\hline & HIIT & AET & & \\
\hline \multicolumn{5}{|l|}{ DFS (kg) } \\
\hline Before & $39.05 \pm 7.61$ & $35.82 \pm 7.48$ & 18.098 & $0.001^{*}$ \\
\hline After & $41.68 \pm 6.95$ & $40.07 \pm 7.46$ & & \\
\hline F-value & 0.994 & & & \\
\hline$p$-value & 0.330 & & & \\
\hline \multicolumn{5}{|l|}{ DFS/kg } \\
\hline Before & $0.53 \pm 0.09$ & $0.46 \pm 0.09$ & 17.787 & 0.001 * \\
\hline After & $0.56 \pm 0.09$ & $0.52 \pm 0.09$ & & \\
\hline F-value & 0.444 & & & \\
\hline p-value & 0.512 & & & \\
\hline \multicolumn{5}{|l|}{ PFS (kg) } \\
\hline Before & $31.21 \pm 5.68$ & $29.04 \pm 4.88$ & 23.645 & $0.001^{*}$ \\
\hline After & $38.86 \pm 7.76$ & $33.56 \pm 6.15$ & & \\
\hline F-value & 1.558 & & & \\
\hline p-value & 0.226 & & & \\
\hline \multicolumn{5}{|l|}{ PFS/kg } \\
\hline Before & $0.43 \pm 0.08$ & $0.38 \pm 0.07$ & 22.506 & 0.001 * \\
\hline After & $0.53 \pm 0.12$ & $0.44 \pm 0.08$ & & \\
\hline F-value & 1.900 & & & \\
\hline p-value & 0.183 & & & \\
\hline
\end{tabular}

HIIT: high intensity interval training, AET: aerobic exercise training, DFS: dorsi flexors strength, PFS: plantar flexors strength, SD: standard deviation.

*significant differences.

grams of the HIIT group (12 subjects) and the AET group (11 subjects), respectively, completed all 24 exercise sessions. The ankle muscle strength and ratio of the subjects after exercise were significantly greater than those before exercise in both the HIIT and AET groups $(\mathrm{p}<0.01)$ (Table 3). However, there was no significant difference in the ankle muscle strength and ratio of the subjects between the HIIT and AET groups $(\mathrm{p}>0.05)$ (Table 3).

\section{DISCUSSION}

In this study, 23 healthy male subjects performed different recumbent cycle exercise programs for 40 minutes/session, three sessions a week, for a total of 24 sessions for two months to compare the ankle muscle strength and ratio between before and after exercise programs and between the two groups (HIIT vs. AET)

The study hypotheses of this study were, first, "two recumbent cycle ergometer training methods, HIIT and AET, will contribute to the improvement of ankle muscle strength and ratio after exercises compared to before exercises", and second "The subjects assigned to the groups for the two types of training programs will have different improvements in ankle muscle strength." As a result of this study, the first study hypothesis was supported, but the second study hypothesis was not supported.

In this study, based on previous studies indicating that not only recumbent cycle exercise, which involves pedaling in a recumbent position, is recommended as an exercise therapy program for heart failure patients because it has a lower cardiac burden than pedaling in an upright sitting position. ${ }^{3,4}$ But it also has large effects in improving daily living activity functions because the muscle contraction pattern of the lower limb is similar to that of standing up and stair climbing. ${ }^{1}$ In addition, the lower cardiac burden in a recumbent position may have contributed to the improvement of ankle muscle strength while both the HIIT and the AET group were performing the exercises. Both HIIT and AET can contribute to the improvement of both DFS and PFS and ratio after the exercise programs. ${ }^{6,12}$ In addition, individuals can hardly continue AET at speeds not lower than 60 RPM because of the nature of recumbent cycle exercise that leads to muscle fatigue quickly due to the constant and repetitive muscle contractions of the lower limb extensor. ${ }^{1,2}$ Therefore, the HIIT exercise program in which the pedals are turned at a low speed of 20 RPM, provided that they are repeated with low loads and high loads as an exercise method, may be suitable for the elderly generation.

In previous studies on recumbent cycle ergometer training and lower body function improvement, 12 male subjects with severe heart failure ( $57.5 \pm 0.8$ years) performed exercise training composed of 20 minutes of HIIT and 20 minutes of AET for 40 minutes/session for a total of 24 sessions. The isometric maximum strength of the knee joint extensor increased $18 \% .{ }^{4}$ However, the difference between the exercise methods could not be identified in the previous study because two exercise training programs were applied in combination. In this study, when 23 healthy normal subjects were divided into two groups according to exercise method and performed training, all variables of ankle muscle strength significantly increased after training compared to before training.

However, the differences between the two training programs using the recumbent cycle were not significant in all variables of the ankle muscle strength and ratio in the two groups. These results suggest that the HIIT method can provide similar effects to those of AET as an exercise program for the prevention of falls of the subjects who can hardly maintain a pedaling speed of 60 RPM or higher. In addition, in the results of this study conducted with active subjects, it may be expected that the HIIT method can provide relatively similar effects to increase ankle muscle strength as the AET method. 
In ankle muscle strength, PFS affects walking speed and dynamic balance ability regardless of age. In a systematic review, ankle PFS had significant positive correlations with walking speed $(r=0.34)$ and dynamic balance $(r=0.42){ }^{7}$ In addition, a previous study investigated lower extremity muscle strength and dynamic balance in the elderly. ${ }^{13}$ it was reported that knee joint flexor strength and PFS in 35 women (68.1 \pm 4.8 years) and 14 men (71.2 \pm 7.7 years) had significant correlations with dynamic balance ability when going down stairs. Since PFS affects balance regardless of age, a decrease in ankle muscle strength due to aging may further increase the risk of falls. A previous study compared an elderly group aged at least 65 years and two young age groups (20-40 years, 41-65 years) and according to the results, isometric maximal DFS significantly decreased in the elderly group, and dynamic balance ability also significantly decreased in the elderly group. ${ }^{6}$

The results of this study and previous studies identified that recumbent cycle exercise had significant training effects on the strength of ankle muscles among lower limb muscles. Given these results, it was identified that the effects of recumbent cycle exercise to strengthen both DFS and PFS and ratio were similar regardless of whether the HIIT method or the AET method was used, and the rate of increase in DFS was higher when the HIIT method was used. Given these results, the HIIT program, in which the pedals are turned at a low speed, enables the achievement of similar or greater effects in increasing ankle muscle strength compared to AET, in which the pedals are turned at a standard and constant speed of 60 RPM. Therefore, the HIIT program can be a recommendable training method for those elderly persons who refrain from exercise due to weak cardiopulmonary fitness.

There are limitations to this study. First, the small sample size that participated in the recumbent cycle exercise programs. Further study and a larger sample size are necessary. Second, only male subjects participated, so generalization of the study findings is limited. Third, variable functional effects such as balance ability and gait pattern were not included. Fourth, a surface electromyogram (EMG) was not used for the ankle muscle activities. Further study is necessary with the surface EMG to investigate ankle muscle activities.

In clinical conclusion, the HIIT and AET exercise programs were performed for 40 minutes/session, three sessions a week, for a total of 24 sessions. First, recumbent cycle ergometer training significantly increased the subject's PFS and DFS compared to those before exercise with both training methods. Second, the HIIT and AET programs using the recum- bent cycle showed no significant difference in PFS and DFS between the two groups that underwent their respective training programs.

Based on these findings, to increase PFS and DFS using the recumbent cycle, the HIIT method (high-load low-speed pedaling exercise) can be recommended as a safer exercise than the AET method (low-load highspeed pedaling exercise) because it can reduce the frequency of use of joints and insufficient cardiopulmonary capacity.

\section{REFERENCES}

1. Kerr A, Rafferty D, Moffat F et al. Specificity of recumbent cycling as a training modality for the functional movements; sit-to-stand and stepup. Clin Biomech. 2007;22(10):1104-11.

2. Egaña M, O'Riordan D, Warmington SA. Exercise performance and $\mathrm{VO} 2$ kinetics during upright and recumbent high-intensity cycling exercise. Eur J Appl Physiol. 2010;110(1):39-47.

3. Ryu HY, Lee JH, Youn JC et al. Comparison of cardiopulmonary parameters between recumbent and upright cycle in healthy subjects: clinical application for heart failure rehabilitation program. J Korean Acad Cardiorespir Phys Ther. 2014;2(1):31-4.

4. Song JS, Ryu HY, Kang SM. The effect and safety of recumbent cycle exercise in patients with heart failure with reduced ejection fraction. J Korean Acad Cardiorespir Phys Ther. 2020;8(1):19-25.

5. Gregor SM, Perell KL, Rushatakankovit S et al. Lower extremity general muscle moment patterns in healthy individuals during recumbent $c y-$ cling. Clin Biomech. 2002;17(2):123-9.

6. Bok SK, Lee TH, Lee SS. The effects of changes of ankle strength and range of motion according to aging on balance. Ann Rehabil Med. 2013; 37(1):10-6.

7. Oskouei ST, Malliaras P, Jansons P et al. Is ankle plantar flexor strength associated with balance and walking speed in healthy people? a systematic review and meta-analysis. Phys Ther. 2021;101(4):pzab018.

8. Gregor RJ, Fowler EG. Biomechanics of cycling. In: Zachazewski J, eds, Athletic Injuries and Rehabilitation, Philadelphia, WB Sanders, 1996:367-88.

9. Fregly BJ, Zajac FE. A state-space analysis of mechanical energy generation, absorption, and transfer during pedaling. J Biomech. 1996;29(1): $81-90$.

10. Kato M, Tsutsumi T, Yamaguchi T et al. Characteristics of maximum performance of pedaling exercise in recumbent and supine positions. J Sports Sci Med. 2011;10(3):491-7.

11. Walsh-Riddle M, Blumenthal JA. Cardiovascular responses during upright and semi-recumbent cycle ergometry testing. Med Sci Sports Exerc. 1989;21(5):581-5.

12. Buckinx F, Croisier JL, Reginster JY et al. Reliability of muscle strength measures obtained with a hand-held dynamometer in an elderly population. Clin Physiol Funct Imaging. 2017;37(3):332-40.

13. Kovacikova Z, Sarvestan J, Neumannova K et al. Balance control during stair descent on compliant surface is associated with knee flexor and ankle plantar flexor strength in older adults. J Biomech. 2020;111:110013. 